\title{
ROBTK: An intelligent robot to transport objects
}

\author{
Wallyson O. das Mercês ${ }^{1}$, Edson M. da Costa ${ }^{2}$, Jaílton W. Tavarez ${ }^{1}$, \\ Patrícia P. Diniz ${ }^{1}$, Renato H. Torres ${ }^{3}$
}

${ }^{1}$ Instituto Federal de Educação, Ciência e Tecnologia do Pará (IFPA) - Paragominas, PA - Brasil

${ }^{2}$ Departamento de Sistemas e Computação

Universidade Federal Rural da Amazônia (UFRA) - Paragominas, PA - BRasil

${ }^{3}$ Instituto Federal de Educação, Ciência e Tecnologia do Pará (IFPA) - Vigia, PA - Brasil

\{wallyson.om, edsonmagalhaesdacosta\}@gmail.com, renato.hidaka@ifpa.edu.br

\begin{abstract}
Studies show that every year the number of accidents and medical care expenses increase due to the work accident. In the context of transporting objects, many activities are still carried out by human labor which in many cases may be subject to disasters due to the high number of hours worked or due to unhealthy activities. Taking into account the presented scenario, this work aims to introduce the robot ROBTK an intelligent robot to transport objects. We developed robot intelligence building a convolutional neural network $(C N N)$ to perform object classification. The tests performed showed that both the intelligence and the mechanics designed are efficient.
\end{abstract}




\section{Introduction}

According to the Digital Observatory of Health and Safety of Labor [Smartlab 2016], between 2012 to 2016, more than 3 million work-related accidents occurred throughout Brazil. Of the registered causes, cuts $(21.11 \%)$, fracture $(17.49 \%)$ and contusion $(15.86 \%)$ are the top-3 causes of work accidents observed. The observatory also noted that, in the same period, the industries lost the equivalent of more than 254 million working days due to social security. This amount reflected in an expense of more than 20 billion reais. To reduce accidents and labor costs, factories or suppliers that work with the transport of material are increasingly investing in robotic solutions.

Considering the importance of this topic, in 2015, Amazon held the First Amazon Picking Challenge [Correll et al. 2018]. The challenge goal was to design an autonomous robot to pick items from a warehouse shelf. Human workers currently perform this task, and there is hope that robots can someday help increase efficiency and throughput while lowering cost. Inspired by the Amazon Picking Challenge, we propose the robot ROBTK that is a robot intelligent for the transport of objects. We built a convolutional neural network $(\mathrm{CNN})$ to recognize and classify transported objects. Depending on the classification, ROBTK plans the route to transport the object to the right place.

To prove the efficiency of the solution, the robot was trained to recognize pieces of the chessboard. Depending on the recognition, the ROBTK has to take the piece to the pre-defined location. Considering the autonomy of the ROBTK, it could be used in environments of transport of materials of high risk for human health, stock control or civil construction.

\section{Methodology}

\subsection{Convolutional Neural Network}

$\mathrm{CNN}$ is a type of deep-learning that became popular due to its excellent performance in visual objects recognition and classification problems. According to [Goodfellow et al. 2016], a convolutional layer is formed by 3-tuple $<$ convolution, activation function, pooling $>$. Convolution works as feature maps. Each convolution has the function of learning the specific characteristics of the input data. The activation functions are responsible for the nonlinearity of the model. In the context of the CNN, studies such as [Gu et al. 2018] and [LeCun et al. 2015] point out that the Rectified Linear Unit $(\mathrm{ReLu})$ function and its variations have good performance for the neurons of the convolution layers. The pooling function, the last component of 3-tuple, has the goal of merging semantically similar features and make feature mapping invariant.

Figure 1 shows the CNN developed in this work. We use skit-learning and Keras APIS to build this CNN. The network has three convolutional layers and six neurons in the output layer. The six neurons of the output layer are justified because the problem we want to solve has six classes: $\{\mathrm{C} 0$ : king, $\mathrm{C} 1$ : queen, $\mathrm{C} 2$ : pawn, C3: horse, C4: tower, C5: bishop\}.

We have set aside two-third of the dataset to performing the training and validation of the model using holdout method. In the two-thirds of the dataset, each class has 31 samples. To ensure the stability of the model, we repeated ten times the process of the holdout. After training and validation, we used a third of the remaining data to estimate 


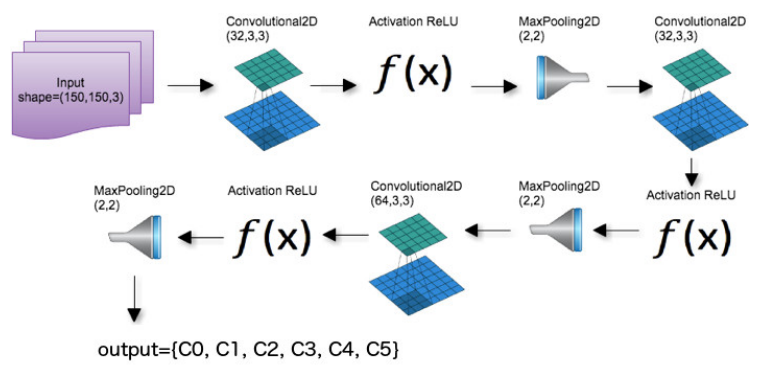

Figura 1. CNN design: The architecture has three convolutional layers with $3 \times 3$ size filters. The first two layers have 32 filters, and the last one has 64 filters.

the generalization performance of the model. Figure 2 shows the learning curve of the training phase, and the confusion matrix calculated from the prediction of the test data. In the learning curve, we can see that the model was able to learn from the training data and also to generalize since it presented high accuracy $(\approx 0.98)$ with the validation data.

The high accuracy and proximity of the training and validation curves shows us that the model does not present overfitting. We confirm this behavior in the confusion matrix of unseen data. In this matrix, we can see that almost all samples are correctly classified. The only exception were two false negatives of the C5 class. Considering the performance analyzed, we can verify that $\mathrm{CNN}$ is efficient to solve the proposed problem.
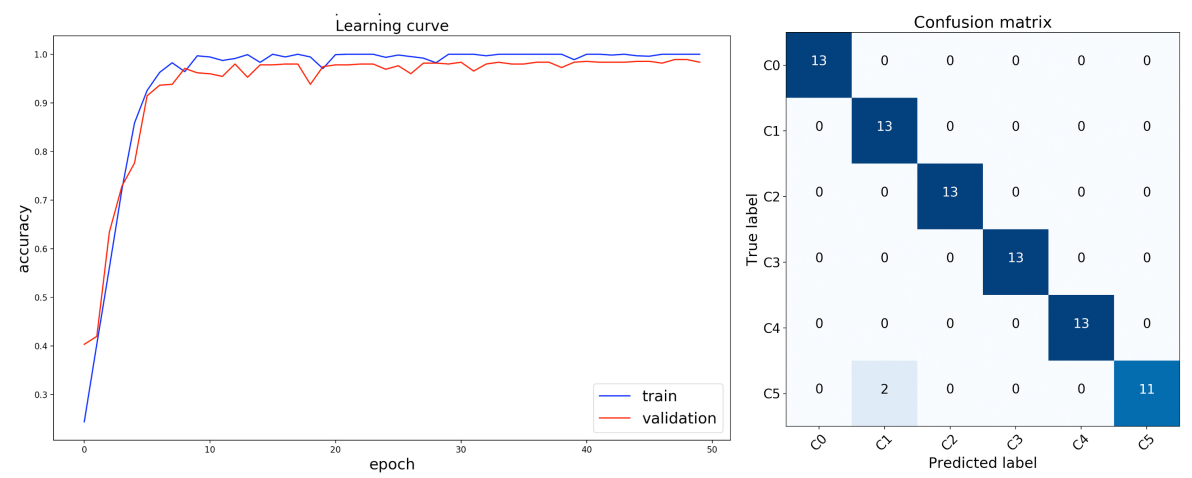

Figura 2. Learning curve and confusion matrix computed in the training and test phase.

\subsection{Robot Construction}

Figure 3 shows the ROBTK and its electrical scheme. ROBTK can be classified as a line follower robot because it uses two reflective optical sensors (item 8) to control its direction. We also use an ultrasonic sensor (item 6) to detect the object, and a raspberry to control the camera and run the neural network. After running CNN, the robotic arm is driven to carry the object. The line circuit that the robot traverses depends on the object being classified.

In our test circuit, the ROBTK follows the path until the ultrasonic sensor detects the object. At this point, the camera captures the image of the object and CNN performs the classification. Then the robotic arm picks up the object and takes it to the pre-defined location. As we can observe in figure 4, each piece has its container defined. We performed 12 tests on the circuit of figure 4, and all tests were successfully performed. 


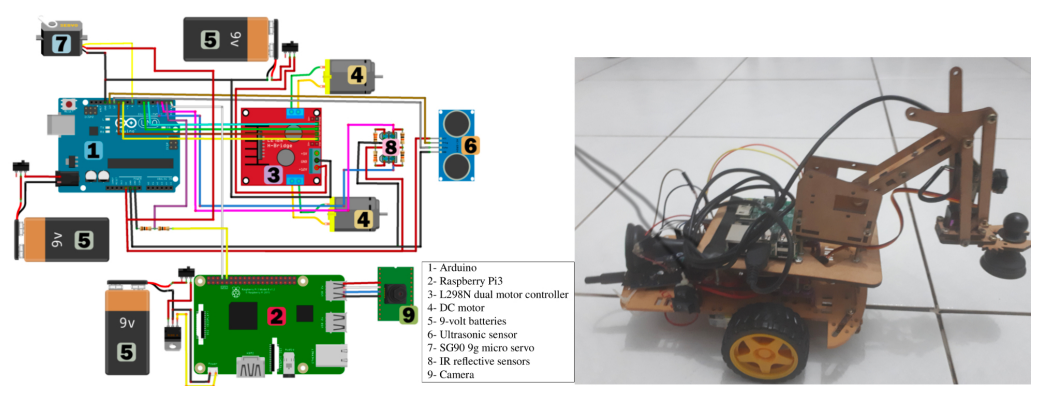

Figura 3. ROBTK electric circuit design.

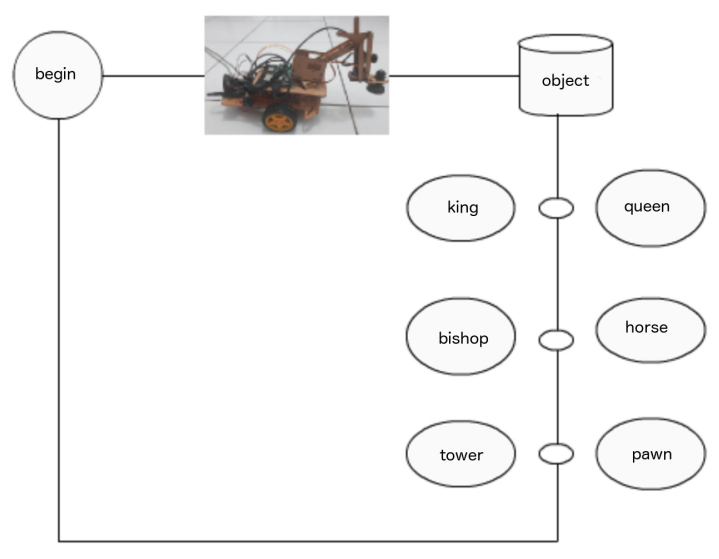

Figura 4. ROBTK test circuit.

\section{Conclusion}

We understand that the use of robots in high-risk environments is essential to avoid workrelated accidents and medical expenses. In this context, we presented the design of an intelligent robot for the transport of objects. In the tests performed, we demonstrate that the intelligence of ROBTK can be used to replace human labor in transporting objects in high-risk environments. In practice, to use ROBTK in different contexts, we would have to retrain $\mathrm{CNN}$.

\section{Referências}

Correll, N., Bekris, K. E., Berenson, D., Brock, O., Causo, A., and and, K. H. (2018). Analysis and observations from the first amazon picking challenge. IEEE Transactions on Automation Science and Engineering, 15:172-188.

Goodfellow, I., Bengio, Y., and Courville, A. (2016). Deep Learning. MIT Press. http: / / www. deeplearningbook.org.

Gu, J., Wang, Z., Kuen, J., Ma, L., Shahroudy, A., Shuai, B., Liu, T., Wang, X., Wang, G., Cai, J., and Chen, T. (2018). Recent advances in convolutional neural networks. Pattern Recognition, 77:354-377.

LeCun, Y., Bengio, Y., and Hinton, G. (2015). Deep learning. Nature, 521(7553):436444.

Smartlab (2016). Observatório nacional de segurança do trabalho. https:// observatoriosst.mpt.mp.br. 